\title{
EL PLAN DE CLASE, UN GÉNERO PROFESIONAL: CÓMO LO NARRAN Y LEGITIMAN LOS PROFESORES NOVATOS DESDE EL PARADIGMA DE LA MULTIMODALIDAD*
}

\author{
THE CLASS PLAN, A PROFESSIONAL GENRE: HOW \\ BEGINNING TEACHERS NARRATE AND LEGITIMATE \\ IT FROM THE MULTIMODALITY PARADIGM
}

\section{BEATRIZ FIGUEROA**, MARIANA AILLON**, STEFFANIE KLOSS ${ }^{* * *}$}

\section{RESUMEN}

El estudio describe, desde un modelo de investigación cualitativa fenomenográfica, cómo un grupo de estudiantes terminales de la carrera de Pedagogía en Español de la Universidad de Concepción percibe la naturaleza y función del plan de clases como género discursivo profesional. En la arquitectura del discurso destaca el hipertexto que opera con un metalenguaje conformado por códigos multimodales, conectando una serie de discursos disciplinarios/pedagógicos en un proceso de compleja intertextualidad. El análisis permite observar la configuración del plan como un activo factor cultural, que hace variar la tradicional interacción simbólica liceo/universidad, resignificando la construcción del fenómeno del aprendizaje, esencia de la praxis docente.

Palabras clave: Plan de clase, género discursivo profesional, hipertexto, intertextualidad.

\section{ABSTRACT}

This study describes from a qualitative phenomenographic research model how a group of students finishing their training program in Spanish Teaching at the Universidad de Concepción, Chile, perceives the nature and function of the class plan as a professional

* La investigación forma parte del Proyecto Fondecyt Regular № 1110909 “Alfabetización académica: el hipertexto una herramienta para mejorar los aprendizajes de profesores".

** Doctora en Educación e Investigadora Responsable del proyecto Fondecyt N ${ }^{\circ} 1110909$. Universidad de Concepción. Concepción, Chile. Correo electrónico: bfiguero@udec.cl

*** Magíster en Literaturas Hispánicas y Doctoranda en Lingüística. Universidad de Concepción. Concepción, Chile. Correo electrónico: maillon@udec.cl

$\star * \star *$ Profesora de Español y estudiante de Magíster en Lingüística Aplicada. Universidad de Concepción. Concepción, Chile. Correo electrónico: steffaniekloss@udec.cl 
discursive genre. In speech framework, hypertext stands out, which operates with a metalanguage made up of multimodal codes, linking a series of pedagogic/disciplinary speeches in a process of complex intertextuality. The analysis enables us to see the plan configuration as an active cultural factor that makes the traditional symbolic schooluniversity interaction change, thus providing a new meaning to the construction of the learning phenomenon, which is the essence of the teaching praxis.

Keywords: Class plan, professional discursive genre, hypertext, intertextuality.

Recibido: 08.11.12. Aceptado: 22.01.13.

\section{INTRODUCCIÓN}

U

N GÉNERO DISCURSIVO académico es la estructura retórica que utilizan los profesionales para solventar buena parte de las tareas o de las actividades que se deben resolver en el ámbito laboral. Cada disciplina posee su propio repertorio de géneros discursivos, armonizados según sus necesidades y prácticas sociales (Cassany, 2006).

Para llevar a cabo su acción profesional, el profesor requiere elaborar un plan de clases que le sirva como mapa de ruta de su trabajo en el aula. A través de esta programación describe, desde las perspectivas de los objetivos de aprendizaje, las actividades que se ejecutarán para lograr las metas propuestas. Partimos de la premisa de que toda producción textual, junto con cumplir con una dimensión discursiva pragmática posee una dimensión epistémica, generadora de conocimiento. De esta forma, el interés por estudiar el género de este texto radica en que al describir cómo es, cómo funciona y cómo es percibido por quienes lo utilizan, podemos mejorar su aprendizaje, considerando que aprender a producirlo implica un manejo de las prácticas profesionales que se conciben en torno a él, en el sentido de que "el discurso desempeña un papel esencial en una organización como entidad; a través de éste construye y mantiene la imagen de dicho sistema. Desde el contenido y la forma del discurso una organización recuerda su historia, crea visiones del futuro y actualiza sus objetivos, procedimientos e ideas" (Gunnarsson, 2003, p. 18).

Objetivo del estudio: La investigación describe de qué forma construye el plan de clases y cuáles son las percepciones que tiene de él un grupo de estudiantes terminales de pedagogía. Además, se busca identificar aportes significativos de la formación disciplinario/pedagógica que tributen al desarrollo de esta escritura académica, fundamentalmente desde dos perspectivas: las dimensiones cognitiva y social. 
Metodología: La investigación, de tipo cualitativo descriptivo, se ciñe al modelo fenomenográfico creado por Marton y Säljö (1976), a propósito del estudio de los aprendizajes de calidad en el área del lenguaje. La fenomenografía se preocupa por describir, desde la perspectiva del aprendizaje, los cambios de conciencia ocurridos en los estudiantes cuando experimentan el mundo que los rodea (Marton y Booth, 1997).

La teoría de la variación fenomenográfica permitió, por una parte, describir los aspectos comunes de las percepciones de los estudiantes respecto del plan de clases y de esta forma en una primera instancia, configurar la esencia del fenómeno en estudio. Luego, desde esta misma interacción, se identificaron los aspectos variantes o diferentes en las planificaciones elaboradas por aquellos sujetos que logran el mejor manejo del género, puesto que, la variación en los modos de vivenciar los fenómenos de la realidad es vista según la perspectiva fenomenográfica como una condición natural del aprendizaje de calidad.

Por lo tanto, cualitativamente nos preocupamos por establecer las categorías emergentes de ambos procesos, las identificadas como comunes, así como aquellas consideradas como manifestación de la variación. Sin embargo, el estudio privilegió el reconocimiento de estas últimas categorías, considerando que los aspectos relacionados con los aprendizajes de calidad en las tareas de elaboración del plan de clases constituyen los insumos significativos que interesan en la descripción del fenómeno.

Muestra del estudio: Se trabajó con una muestra de 25 estudiantes de la carrera de Pedagogía en Español de la Universidad de Concepción, los que durante el primer semestre del año 2011 desarrollaron su práctica profesional en diferentes establecimientos de la comuna de Concepción y participaron de la asignatura de Taller de Especialidad. Con ambas actividades, este grupo concluyó su formación pedagógica.

Los datos fueron recogidos a través de un cuestionario y de entrevistas semiestructuradas. Además, se recolectaron planificaciones elaboradas por los sujetos en el transcurso de su práctica profesional, en el marco de la asignatura Taller de Especialidad. Con los dos primeros instrumentos se recopiló información respecto de la percepción que tenían los estudiantes sobre el plan de clases, abordando aspectos relacionados con los procesos de elaboración de las planificaciones, las fortalezas y debilidades en la ejecución, grado de funcionalidad del texto como apoyo a las tareas pedagógicas, entre otros. Las planificaciones fueron utilizadas para triangular la información obtenida de los cuestionarios y las entrevistas. 


\section{NATURALEZA Y FUNCIÓN DEL PLAN DE CLASES}

Según la percepción de los estudiantes, el plan de clases es un género que refleja las competencias adquiridas en el transcurso del proceso de formación de la carrera docente. Constituye una síntesis del saber pedagógico en la que se articulan conocimientos disciplinarios con una perspectiva didáctica que señala las directrices de cómo se debe asumir la enseñanza de ciertos contenidos. Uno de los comentarios del Sujeto 5 ilustra esta afirmación:

La planificación es la principal herramienta de los profesores, nos brinda seguridad y nos ayuda a optimizar de mejor forma los recursos. Es una ayuda ciento por ciento necesaria. Una guía y una obligación, ya que sin ella realmente, el improvisar nos pasaría la cuenta, ya que no estarían sistematizados los aprendizajes, no habría coherencia y todo sería un desastre, tanto para uno como para los alumnos. (Sujeto 5).

Los estudiantes también consideran el plan de clases como una herramienta que define el saber hacer docente y que les permite como profesores noveles participar en la cultura discursiva profesional, dialogando con los distintos agentes del sistema: profesores, directivos, supervisores.

Las planificaciones además de ser revisadas por el profesor guía fueron leídas por el jefe técnico del liceo que varias veces después de la ejecución me preguntó cómo me había resultado alguna de las actividades descritas en especial respecto de los logros alcanzados. (Sujeto 8).

El plan presenta el diseño de una o un conjunto de clases para alcanzar, de manera secuenciada, determinados aprendizajes. Por tanto, delimita los núcleos básicos del aprendizaje, los gradúa, sistematiza y organiza en articulación con las estrategias que permitan alcanzarlos. La programación se llevará a cabo a partir de una secuencia de actividades distribuidas en espacios y tiempos determinados y de acuerdo al uso de recursos específicos.

Esta programación además de ser un mapa de ruta, es un referente que permite la reflexión metacognitiva del docente luego de su experiencia de ejecución. Promueve, por tanto, el avance del conocimiento profesional experimental.

Después de hacer la clase es bueno el análisis, anotar como bitácora lo que resultó y qué aparte de lo planeado es necesario mejorar o cambiar, si faltó algún material, si fue atractivo para los alumnos. Una cosa es cómo uno se imagina que ocurrirá la clase, luego cómo ocurrió realmente es otra. (Sujeto 4). 
Desde la perspectiva sociocultural, el plan forma parte del protocolo de comunicaciones de la comunidad educativa. Sus autores lo escriben como referente para sí mismos y además, para informar de su trabajo a los pares y directivos de sus unidades. Es un texto que oficializa la programación del aula a través del cual la dirección de una unidad educativa cautela que el currículum se implemente contextualizado a la realidad local y de acuerdo a los parámetros de calidad establecidos por las políticas educativas nacionales. En el testimonio siguiente se observa esta preocupación del centro por velar que las planificaciones se ajustaran a los requerimientos institucionales:

Mis planificaciones por unidades abarcaban de doce a dieciocho horas pedagógicas, mi profesor guía las revisaba y luego yo las ajustaba con sus sugerencias. Esta tarea era importante porque luego las revisaba la directora del departamento de lenguaje del colegio para ponerle el timbre y dejarlas registradas. (Sujeto 7).

\section{ESTRUCTURA Y ELEMENTOS QUE COMPONEN EL GÉNERO DISCURSIVO}

La planificación se compone de una articulación coherente de los elementos curriculares que intervienen en el proceso de enseñanza: objetivos, contenidos, actividades y evaluación. El formato más tradicional en nuestro país es una tabla que se organiza en columnas, cada una de las cuales considera los elementos señalados anteriormente y en la que se usan ciertas formas discursivas particulares. La primera columna, de izquierda a derecha, presenta los objetivos o aprendizajes esperados, los que describen las metas enunciándolas mediante un verbo que señala una conducta susceptible de evaluar. La segunda columna indica el contenido o materia que contextualiza la planificación; la tercera designa las actividades a partir de las cuales los estudiantes alcanzarán los objetivos. Esta columna constituye una secuencia narrativa que en forma sintética señala las tareas a desarrollar, por los estudiantes, según la fase de la clase. Finalmente, la última columna en el lado derecho de la tabla, presenta los criterios, procedimientos e instrumentos con los cuales serán evaluados los objetivos (Tabla 1):

Es un texto que al comienzo no es fácil de redactar, es como poner por escrito la escena de cómo uno se imagina ocurrirá la clase. Hay que aprender a usar los verbos en la redacción de los objetivos y la forma en que se redactan las actividades, cada columna se articula con la anterior. (Sujeto 4). 


\begin{tabular}{|c|c|c|c|c|}
\hline Nivel: NM3 & \multicolumn{4}{|c|}{$\begin{array}{c}\text { Subunidad 1: El viaje como tema literario } \\
\text { Tiempo: } 1 \text { hora ( } 45 \text { minutos) }\end{array}$} \\
\hline OFT: & \multicolumn{4}{|c|}{ Desarrollo del pensamiento: análisis, interpretación y reflexión textual. } \\
\hline UNIDAD & CONTENIDOS & $\begin{array}{c}\text { APRENDIZAJE } \\
\text { ESPERADO }\end{array}$ & ACTIVIDADES & EVALUACIÓN \\
\hline $\begin{array}{l}\text { La literatura } \\
\text { como } \\
\text { fuente de } \\
\text { argumentos, } \\
\text { modelos } \\
\text { y valores } \\
\text { para la vida } \\
\text { personal y } \\
\text { social. }\end{array}$ & $\begin{array}{l}\text { Diversas } \\
\text { formas del } \\
\text { viaje en la } \\
\text { tradición } \\
\text { literaria: } \\
\\
\text { - Viaje a los } \\
\text { infiernos. }\end{array}$ & $\begin{array}{l}\text { Reconocen } \\
\text { la forma de } \\
\text { viaje a los } \\
\text { infiernos en } \\
\text { la literatura, } \\
\text { atendiendo } \\
\text { a la } \\
\text { forma y el } \\
\text { significado } \\
\text { del texto. }\end{array}$ & $\begin{array}{l}\text { Fase I: Presentación de los contenidos } \\
\text { Motivación. } \\
\text { Los estudiantes observan un video sobre el viaje a } \\
\text { los infiernos. Posteriormente, el docente propicia } \\
\text { la reflexión respecto al tema del viaje. } \\
\text { Activación de conocimientos previos. } \\
\text { Los alumnos dialogan sobre el viaje, entendiendo } \\
\text { este como un proceso de cambio. } \\
\text { Presentación de los contenidos. } \\
\text { Los alumnos observan un power point que } \\
\text { contextualiza el tema del viaje. Esta presentación } \\
\text { se centra en los siguientes conceptos: } \\
\text { - El viaje considerado como un cambio de estado. } \\
\text { - Representación del viaje a través de la literatura. } \\
\text { - Viaje a los infiernos. } \\
\text { Materiales: video y power point sobre los } \\
\text { conceptos claves del tema del viaje. } \\
\text { Tiempo: } 10 \text { minutos } \\
\text { Fase II: Desarrollo de los contenidos. } \\
\text { Los alumnos reciben una guía con extractos } \\
\text { de la Divina Comedia, mediante el cual deben } \\
\text { identificar la forma de viaje a los infiernos e } \\
\text { interpretar el contenido y la forma del texto. } \\
\text { Una vez realizada la guía en parejas, se propiciará } \\
\text { la reflexión del curso, enfatizando en los siguientes } \\
\text { aspectos: } \\
\text { - Tema del viaje a los infiernos } \\
\text { - Interpretación del texto mediante su forma } \\
\text { literaria } \\
\text { Tiemplema a Interiales: Cuaderno y lápices de colores } \\
\text { Elatel minutos. } \\
\text { Materiales: guía de trabajo } \\
\text { Tiempo } 25 \text { minutos. } \\
\text { Fase III: Síntesis y transferencia } \\
\text { Los alumnos realizan un esquema en su cuaderno } \\
\text { que sintetice el fragmento leído. El esquema debe } \\
\text { considerar: } \\
\text { 1. Definición del viaje y la forma en que lo aborda } \\
\text { la literatura } \\
\text { 2. El viaje a los infiernos y la ejemplificación del } \\
\text { concepto. } \\
\text { 3. Jerarquización de las ideas expresadas } \\
\end{array}$ & $\begin{array}{l}\text { Procedimiento } 1 \\
\text { Video de un extracto } \\
\text { de la Divina Comedia. } \\
\text { Evaluación: } \\
\text { Formativa. } \\
\text { Instrumento: Sin } \\
\text { instrumento de } \\
\text { evaluación. } \\
\text { Indicadores: } \\
\text { Los alumnos y } \\
\text { alumnas } \\
\text { - Definen el concepto } \\
\text { de viaje. } \\
\text { - Relacionan el } \\
\text { tema del viaje en la } \\
\text { literatura con su vida } \\
\text { cotidiana } \\
\text { Procedimiento 2: } \\
\text { Guía de trabajo con } \\
\text { extractos de la Divina } \\
\text { Comedia. } \\
\text { Evaluación: Formativa } \\
\text { Instrumento: Sin } \\
\text { instrumento de } \\
\text { evaluación. } \\
\text { Indicadores: } \\
\text { Los alumnos y } \\
\text { alumnas } \\
\text { - Señalan las } \\
\text { características del } \\
\text { tema del viaje a los } \\
\text { infiernos. } \\
\text { - Interpretan el texto a } \\
\text { través de su estructura. } \\
\text { Procedimiento } 3 \\
\text { Esquema sobre viaje a } \\
\text { los infiernos. } \\
\text { Evaluación: } \\
\text { Formativa. } \\
\text { Instrumento: Sin } \\
\text { instrumento de } \\
\text { evaluación. } \\
\text { Indicadores: } \\
\text { Los alumnos y } \\
\text { alumnas } \\
\text { - Explican la noción } \\
\text { a través de la lectura } \\
\text { de la obra literaria } \\
\text { - Sintetizan los } \\
\text { contenidos aprendidos } \\
\text { - Redactan sus ideas } \\
\text { de forma coherente y } \\
\text { cohesiva }\end{array}$ \\
\hline
\end{tabular}

Tabla 1. Esquema de Subunidad 1. 
Para elaborar el plan ajustado a la organización de los elementos anteriormente señalados, el profesor debe manejar el currículum y, desde este, el conocimiento de los contenidos temáticos. Luego, a partir de la transposición didáctica, adapta los contenidos a los receptores de su plan, transformándolos didácticamente en conocimiento susceptible de ser enseñado.

La clase fue planificada según el modelo visto en las asignaturas de didáctica. Este formato contempla los contenidos, aprendizajes, las estrategias metodológicas y de evaluación, y los materiales y la evaluación. Tiene más detalle y es exigente en la acotación de los aprendizajes, la prefiero porque cautela con mayor precisión que las cosas resulten. (Sujeto 6).

El plan puede adquirir diversos formatos de organización de los elementos en la página, sin que estas diferencias afecten su composición semántica y/o pragmática. En cuanto a extensión, en el sistema educativo, los requerimientos varían desde planes muy amplios semestrales, trimestrales o mensuales, hasta otros muy ajustados, clase a clase. Una secuencia amplia y general no permite entrar en detalles respecto a la progresión didáctica de los aprendizajes, mientras que un plan de menor extensión posibilita la descripción de un aprendizaje acotado, especifica un foco de desarrollo y orienta la secuencia de actividades desde lo más simple a lo más complejo, equilibrando las dimensiones teórico/prácticas del diseño en cuestión. El siguiente enunciado da cuenta de esta diferencia:

En la planificación clase a clase del liceo debo indicar el inicio, desarrollo $y$ cierre de cada clase; las planificaciones elaboradas en la universidad empleaban otra taxonomía: presentación de los contenidos -que incluye una fase de motivación, de activación de conocimientos previos y de presentación de los contenidos propiamente tal- desarrollo de los contenidos y síntesis y transferencia. (Sujeto 1).

En síntesis, la escritura del plan es concebida por los estudiantes como la construcción del saber pedagógico, un tejido de elementos que tiene referentes textuales tomados del currículum, de textos teóricos disciplinarios, de textos didácticos y por último de textos funcionales a partir de los cuales se modela y ejercita la competencia de lenguaje en desarrollo. 


\section{LA INTERTEXTUALIDAD Y EL HIPERTEXTO: DOS ELEMENTOS FUNDAMENTALES EN LA CONSTRUCCIÓN DEL PLAN}

Del análisis cualitativo del discurso destacan dos elementos que determinan la producción del plan: la intertextualidad y el hipertexto. Como ya se señaló, los estudiantes recurren a sus conocimientos disciplinario/didácticos en el proceso de composición del plan, seleccionando los elementos que componen su tejido textual. Se trata de una etapa de búsqueda de información en la que enfatizan los procesos de construcción intertextual, ejercicio que realizan conscientemente extrapolando un camino que disciplinariamente aprendieron en las tareas de análisis de los textos literarios, donde tuvieron que hacer dialogar los textos entre sí. El Sujeto 15 da cuenta de este hecho:

La búsqueda de información tiene su dificultad, en primer lugar está el problema de las fuentes, me preocupo de entrar a sitios confiables según el tema, luego, relaciono las ideas de los distintos sitios, juntando para crear lo que necesito. Por ejemplo, si busco una estrategia de aprendizaje seguro que no la encuentro presentada con el contenido que necesito, pero yo la adapto, al revés encuentro el contenido pero no la forma de ejecutarlo. Es lo mismo que cuando en literatura un marco me sirve para leer distintos textos. (Sujeto15).

La configuración específica que logra el dialoguismo en el texto ha sido denominado como "intertextualidad", con la clara intención de reflejar ese diálogo que se produce en el interior de un enunciado, discurso o texto entre el Sujeto de la enunciación y el Sujeto del enunciado (Kristeva, 1969).

Chartier (2004) afirmaba que la intertextualidad existía en el texto impreso, en las citas, referencias y notas al pie, en un contexto delimitado y definido por el autor, en una unidad reconocible y aprehensible, el libro, como producto terminado. Esa unidad, además, era abordada en un tiempo y un espacio reales (Figueroa et al., 2012); en el contexto tecnológico actual, la intertextualidad es un concepto clave para entender la configuración del hipertexto. Cuando el sujeto está creando su plan, se conecta, a través de enlaces electrónicos (link), con una multiplicidad de fragmentos de textos que son la relación material y explícita entre dichos textos, posibilitada por la red.

Por consiguiente, nos hacemos cargo de que la intertextualidad propiciada en internet marca un cambio profundo en la cultura escrita, lo que tiene por lo menos dos consecuencias clave para las prácticas discursivas que nos ocupan: por una parte, expuesto a un corpus infinito de textos, en 
un espacio y tiempo virtuales, el usuario de la red, en este caso el profesor novato, a partir de su subjetividad, selecciona los enlaces o link, producto de la intuición y/o de la búsqueda informada. La lectura no secuencial le obliga a desempeñar un rol más activo en el camino de la misma, consagrándose al hacer clic en los enlaces, como co-autor del (hiper)texto, que está en proceso de construcción. Por otra parte, a través de los link elegidos, expresión de la intertextualidad, el internauta tiene la posibilidad de producir sus propios textos, como afirma Escolano (2006), y convertirse en un escritor-lector, que

interpreta lo dicho por otros con anterioridad, lector que tiende puentes de intertextualidad porque toma las palabras dichas por otros como referencia a través de la cita, la paráfrasis, la traducción o la transcripción, o bien para dar una respuesta, hacer una réplica o una refutación y una discusión (Castelló, 2007, p. 20).

La construcción del plan de clases como un intertexto se muestra claramente en el testimonio del Sujeto 2:

La dimensión práctica, tal vez, fue dado por las asignaturas de didáctica, pues debíamos construir un marco teórico sólido antes de producir nuestras planificaciones, es decir, crear pero en base a algo preexistente, más bien, tomar la información y moldearla de otra forma, transformarla en actividades, en guías, en discurso, hacer dialogar nuestras planificaciones con las fuentes de información. (Sujeto 2).

Según lo formulado por Iser (2005, p. 224), la 'interpretación' consiste en "traducir algo verbal o no verbal a un registro de naturaleza lingüística", lo que desde el texto analizado en este estudio se traduce en una secuencia práctica de enseñanza. El aprendiz cuenta en este proceso con el marco de referencia propio de su disciplina, el que direcciona la búsqueda en la red y le permite aprovechar cognitivamente las ventajas de la no linealidad de una lectura mediante saltos entre uno y otro link, lo que se constituye en fuente de abastecimiento continuo. Esto responde a un paradigma cibernético de la hermenéutica que se revela como una 'autopoiesis en curso' (Iser, 2005). Esta hermenéutica, desde la perspectiva de la construcción del saber en el currículum, se caracteriza por ser "interactiva, intersubjetiva, de los contenidos transmitidos y de los lenguajes en que se expresa, que es la vía más compatible con la intertextualidad resultante y con las formas intermodales de comunicación" (Escolano, 2006, p. 19).

El hipertexto está presente en el plan en la medida que las relaciones 
de intertextualidad entre los referentes teóricos y prácticos conforman una arquitectura, donde los vínculos de sentido, cruzados por el eje pedagógico, son explicitados, respondiendo a una lógica que privilegia la construcción profesional individual de la práctica docente en una disciplina específica.

\section{LA IMPORTANCIA DE LA MULTIMODALIDAD SEMIÓTICA EN LA CONSTRUCCIÓN DEL PLAN}

Otro elemento que condiciona la construcción del texto desde la percepción de los sujetos, tiene que ver con el papel de internet como potenciadora de la circulación de los discursos. Según los datos aportados por los sujetos de la muestra, la mediatización tecnológica y la multimodalidad de códigos que se suman al código lingüístico también cumplen un rol en la construcción de los significados expresados, los que son interpretados y asumidos como conocimiento social de la comunidad de nuevos profesores.

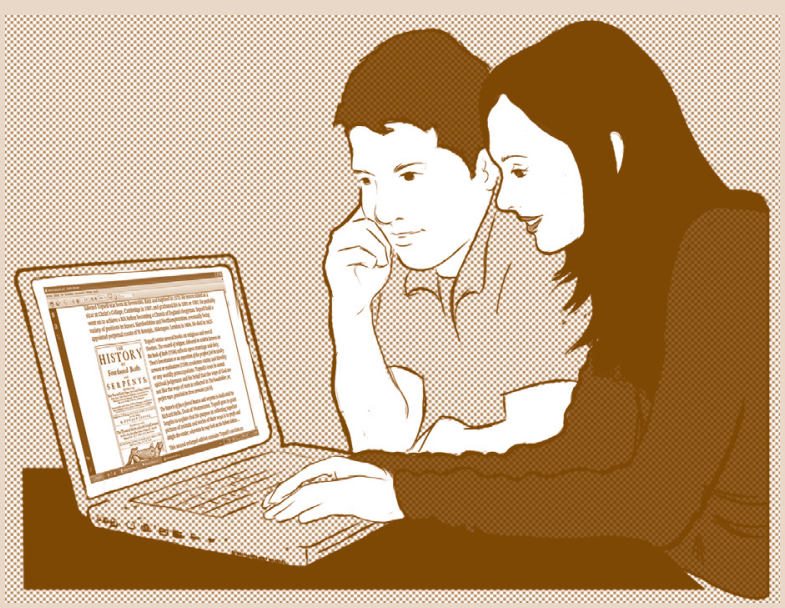

Figura 1.

En el marco de la teoría de la multimodalidad, uno de los recursos semióticos nucleares de los que hace uso el grupo es, precisamente, el acceso a los 'discursos circulantes' (Pardo, 2007). Los estudiantes describen la forma en que acceden a internet ante el desafío común de la elaboración del 
plan de clases, demostrando que el recurso de accesibilidad incluye el conjunto de saberes socialmente distribuidos y las diversas formas en que son apropiados por los miembros del grupo (Pardo, 2007).También influyen las diversas maneras en que se presentan los escenarios de interacción, es decir, el conjunto de objetos portadores de discursos propuestos en diseños específicos e inherentes al acto comunicativo vigente. La socialización del saber y el acceso a la red se puede refrendar en el testimonio siguiente:

(...) el apoyo del grupo fue muy importante en la escritura de la planificación, el grupo de práctica se reúne en el taller una vez a la semana y sabíamos en qué contenido estaba trabajando cada uno, pero siempre estábamos conectados, especialmente por Messenger. Cuando alguno tenía un problema para encontrar material nos ayudábamos a través de la red, compartíamos los sitios web y las bibliotecas virtuales, también compartíamos material didáctico encontrado en el liceo. (Sujeto 18).

Destaca también el hecho de que los sistemas operativos, las aplicaciones de software implicados, las respectivas operaciones indispensables para seleccionar y organizar la información involucrada, configuran patrones de interacción, es decir, se constituyen en visualizadores y objetos semióticos, que apoyan la composición del plan de clases.

Me pasó que tuve que trabajar en medios de comunicación de masas con un texto publicitario y tuve que preparar la edición de un video para ver los estereotipos en la publicidad, no sabía cómo hacerlo, pero mi compañero computin me enseñó, ahora ya no se me olvida". (Sujeto 8).

En consecuencia, la accesibilidad posibilita el uso de recursos semióticos en diversas modalidades y combinaciones, los cuales en la perspectiva de Kress (2003) cumplen el propósito de garantizar la contextualización del discurso en la composición virtual del plan. En este sentido, fijan el conjunto de representaciones derivadas de las creencias, interpretaciones o definiciones intersubjetivas creadas a propósito de la interacción con respecto al ambiente o situación comunicativa. Además, incluyen el acervo de conocimientos históricamente compartidos, así como los roles y las categorías socioculturales desde las que se posicionan los actores sociales y discursivos, junto con el discurso mismo.

Todo lo anterior constituye jerarquías en las que tiene lugar la interacción de la que se nutre el autor, y en las que se activa el cúmulo de saberes pedagógicos para la coordinación de la acción y las cogniciones definidas 
a través de los recursos discursivos. De esta forma, aquí se articulan coherentemente dos factores: de un lado todo lo que implica la accesibilidad y, del otro, el uso consciente y sistemático de los medios tecnológicos para el logro del discurso académico propuesto (Pardo, 2007).

Por tanto, para los jóvenes el significado mediatizado y configurado en el plan de clases, a partir de los recursos de la multimodalidad, pasa a forman parte de su sistema de representaciones de la realidad educativa en el área del lenguaje. Estos recursos multimodales les han posibilitado la percepción de la realidad, a través de los filtros que estructuran los sistemas semióticos, los códigos culturales y los sistemas de representación de las que dispone la comunidad académica profesional a la que se están integrando (Pardo, 2007).

Fue importante para mi haber trabajado revisando lo itemes de la prueba Pisa donde se trabaja con textos discontinuos, donde hay que enseñar a los estudiantes la comprensión de otros códigos además del verbal como lectura de gráficos, mapas, infografías, etc.. Ahí uno se apoya también en los programas computacionales como el prezi”. (Sujeto 12).

Como lo señala (Piscitelli, 2005, p. 84) "las estrategias de trabajo con datos informáticos se vuelven nuevas estrategias cognitivas de trabajo en general y reflejan una lógica social, una ideología y un imaginario de la sociedad".

La constitución de los modelos culturales propios de la era digital ocurre a través de la selección y combinación intertextual, en la que se legitiman formas de comprender y representar realidades sociales. Entonces, el plan de clases se transforma en un discurso que es "producción de producción, experiencia de subjetividad, producción de comunidad y de sentido propio del quehacer docente" (Gunnarsson, 2003, p. 20).

\section{CÓMO SE LEGITIMIZA EL PLAN DE CLASES EN LA COMUNIDAD ACADÉMICA DOCENTE}

$\mathrm{Al}$ avanzar hacia la comprensión del género como parte de la estructura social interesa describir cómo este discurso se legitima dentro de la comunidad a la que representa. En este sentido, adscribimos a la idea de que "la relación del lenguaje y del sistema social no es sólo una relación de expresión, sino una dialéctica natural más compleja en que el lenguaje simboliza 
activamente al sistema social, creándolo y siendo creado por él" (Halliday y Santana, 1982). Desde esta perspectiva afirmamos que los códigos elaborados específicos, como es el caso del plan de clases, poseen un valor de enmarcamiento que los señalan como un medio disponible para institucionalizar y liberalizar los principios dominantes de una entidad en la formación social, como es el caso de la profesión docente en su formación universitaria (Berstein, 2001).

Las interrogantes que nos guían en este apartado tienen que ver con describir cómo el currículum universitario legitima un discurso docente y luego qué impacto tiene este en el sistema escolar en el que se usa. Consideramos siguiendo a Berstein (2001), que la forma que adoptan los códigos elaborados, como es el plan, y su modo de regulación influyen sobre el campo de extensión al que se proyectan, determinando el poder de legitimidad que se les otorga a los miembros de la comunidad que los maneja.

En este sentido, el plan depende de la forma que adquiere en el contexto comunicativo, entendido este como el conjunto de las relaciones sociales que regulan sus posibilidades de comunicación (Berstein, 2001). En el contexto comunicativo, hay dos principios básicos que regulan las posibilidades y el valor de legitimar el género en cuestión: el principio de interacción y el de situación.

El principio de interacción se relaciona directamente con los sujetos de producción y recepción del discurso en cuestión y, entre otras funciones, regula la selección de contenidos, la secuencia de organización de estos, los criterios y el ritmo de su comunicación, junto con la posición y postura de los agentes comunicantes. Por lo anterior, está vinculado con los roles que cumple el estudiante para manejar el género y su uso en un establecimiento.

El principio de situación, en cambio, norma la situación física en donde se produce el discurso y la forma de realización de este, o sea, el conjunto de objetos, atributos, relaciones mutuas y espacio en que estos aspectos se constituyen. Como se observa, considera el discurso en cuanto objeto comunicativo y los aspectos formales que condicionan su proceso de producción como tal (Berstein, 2001).

Los dos principios anteriores determinan las características temporales y espaciales que constriñen el contexto comunicativo del plan de clases. Desde el punto de vista del tiempo, la apropiación del discurso en cuestión se genera en los dos años finales de la formación pedagógica de los docentes, cuando han completado el currículum disciplinario de cuyos contenidos se nutre el plan, y son capaces de proyectarlos en la denominada transposición didáctica. Esta primera aproximación de los estudiantes 
al género plan de clases la calificamos, en palabras de Berstein (2001), de enmarcamiento y elaboración fuerte en la medida en que el transmisor -en este caso, las asignaturas pedagógicas del currículum, que los inician como productores de este tipo de texto- controla, desde su formato, la selección, organización, ritmo y criterios de comunicación, así como también la posición de los comunicantes y la emisión del plan como género. Por tanto, el contexto de interacción comunicativa, es decir, el currículum universitario, define y mandata las reglas específicas para producir un discurso legítimo.

Desde las perspectivas espacial y formal del contexto comunicativo de emisión en la universidad, el desarrollo del género está estrechamente ligado con las dimensiones hipertextuales del discurso, tanto por la multimodalidad de códigos intervinientes, como por la accesibilidad del género, potenciados todos por las herramientas tecnológicas. De esta forma, desde el contexto universitario, el plan de clases se presenta como un género legítimo enmarcado dentro de reglas de reconocimiento en cuanto tal, puesto que los agentes de la comunicación participan conscientemente de ellas y cumplen con el fin comunicativo para lo cual el discurso fue creado.

Sin embargo, cuando en el contexto de la práctica profesional el discurso trasciende el ámbito universitario y pasa a ser reconocido por la comunidad educativa del liceo, las características de la interacción comienzan a variar. En la universidad el discurso es validado por el profesor que revisa el texto, sin probar aún su funcionalidad en la práctica pedagógica. De tal forma, su legitimidad auténtica se alcanzará cuando dentro del enmarcamiento de los poderes académicos del liceo este sea reconocido como funcional. Es decir, cuando pase desde un nivel de elaboración fuerte con foco en el trasmisor (currículum universitario), al contexto de la práctica donde el estudiante o adquiriente tenga un grado de mayor control sobre todos los criterios de organización de su discurso, así como sobre la configuración, organización formal y física del mismo. Por tanto, el nivel de elaboración adquiere mayor autonomía y el estudiante como adquiriente del género pasa a transformarse en trasmisor del mismo. Esta posición de enmarcamiento y elaboración mayor será más fuerte y determinante cuando el nuevo profesor ejerza plenamente su tarea en clases con dominio completo sobre la autorregulación de su práctica pedagógica. En esta etapa final, los principios de contextualización del discurso pasan a establecer las reglas de realización que, en el caso del profesor de aula, legitiman socialmente el género en cuestión. 


\section{CONCLUSIONES}

-Del análisis anterior concluimos que el género examinado delimita una comunidad discursiva con sus normas, conocimientos y prácticas sociales, pues es compartido por la entidad docente que accede a través de él a un conocimiento pedagógico y le reconoce la capacidad de comunicar la dimensión práctica, experimental y reflexiva de la enseñanza.

En la muestra estudiada, las configuraciones espacio-temporales que posibilitan las tecnologías de la información y comunicación aportan nuevas dimensiones al propósito de este género, permitiendo estructurar escenarios educativos más ricos y variados. El nivel de interactividad lleva implícito el cambio del valor de las fuentes de información clásicamente utilizadas, así como también, un cambio de configuraciones vinculares y espacio-temporales en las relaciones y límites del lector/escritor que agencia este género en cuestión (Piscitelli, 2005).

-La organización del plan de clases en contenido y forma es entendida como un discurso que proyecta la manera de vivenciar el fenómeno del aprendizaje, configurando un marco de sentido propio respecto de la enseñanza, en un contexto específico. Como discurso, resulta de la conjunción de la lectura e interpretación del currículum oficial, del manejo de un conocimiento disciplinario y la adscripción a un enfoque didáctico para enfrentar al desafío de la enseñanza, constituyéndose así en la base de la narrativa de los profesores noveles examinados.

-El plan de clases se sitúa como forma retórica enraizada en un ámbito cultural y acoge, desde el conocimiento docente, una determinada mirada de la realidad educativa regional. Como modelo cultural se encuentra fuertemente condicionado por la cultura digital, a través de la cual legitima formas de representar la realidad profesional individual y colectiva, mediatizadas por una variedad de códigos.

-Desde esta perspectiva, es significativo reconocer cómo, a partir del cambio en la redes comunicacionales, el paradigma científico y los sistemas de conocimiento en general, tales como el género, se encuentran a su vez fuertemente entramados con la vida profesional de los sujetos y de la comunidad que lo usa. Todo lo cual conlleva aparejado un cambio epistemológico 
de un conocimiento situado que, a través de las redes interactivas, es beneficiado por un conocimiento multimodal que lo proyecta en una matriz estructural dinámica y fluida.

Lo anterior es el resultado de un proceso dialógico en el que convergen múltiples voces técnicas del tejido discursivo del plan. Dado este dialogismo, el texto no es un recipiente vacío, con un contenido colocado en él desde fuera, sino más bien un generador de ideas pedagógicas. La esencia del proceso de generación no es solo evolución, sino también interacción entre estructuras educativas, como un activo factor cultural que desde un sistema semiótico en acción resignifica el fenómeno del aprendizaje, esencia de la práctica docente (Wertsch, 1993).

-Cabe destacar sin embargo, que el diálogo que origina el texto en cuestión varía con celeridad. En este sentido hay una espiral que, si bien se inicia en el contexto universitario, adquiere forma real y significativa cuando satisface las necesidades concretas de un centro educativo determinado, legitimando así la narrativa de los profesores, en cuanto el discurso escrito se transforma en práctica pedagógica. De esta forma, estas dos comunidades educativas (universidad y liceo) se retroalimentan desde la base del discurso genérico del plan, cumpliéndose así la dialéctica de complejidad del lenguaje (Hallyday y Santana, 1982).

-Como se observa, el texto plan de clases construye y dinamiza las estructuras sociales educativas en la medida que el liceo pasa a convertirse en un gigantesco laboratorio de nuevas posibilidades para la praxis educativa, con lo que constatamos que la universidad ya no es la usina del conocimiento, en términos de saberes socialmente significativos. El código elaborado representado en el plan, con una dimensión de enmarcamiento que varía a lo que el centro considera legítimo y funcional, está avanzando hacia un proceso de liberalización de los principios dominantes que lo condicionaron tradicionalmente. Este discurso posee en la actualidad un efecto regularizador que influye sobre el campo de control simbólico en organismos especializados como es el espacio de interacción: liceo/universidad (Berstein, 2001), modificando las relaciones de reproducción social. En la legitimación del género existe un cambio, la configuración de una relación nueva entre los actores profesionales que lo producen y usan: liceo, universidad, profesores en ejercicio, directivos profesores novatos, entre otros. 
En síntesis, el tipo de arquitectura definida por el hipertexto opera con un metalenguaje conformado por códigos multimodales (escrito, oral y audiovisual), que conecta una serie de discursos, por tanto, de subjetividades que dan origen a una compleja intertextualidad (Colom, 2006). Las voces de los autores y fuentes, cuyos aportes han sido acogidos por el docente que planifica, se pierden en el anonimato, concediendo libertad al intérprete para fundirlas en su propia red de significación y dar sentido así a su quehacer pedagógico. Este es, a nuestro juicio, un paso relevante en la búsqueda por legitimar una nueva praxis discursiva del profesor.

\section{REFERENCIAS}

Berstein, B. (2001). La estructura del discurso pedagógico. $4^{\text {ta }}$ edic. Madrid, España: Morata.

Cassany, D. (2006). Taller de textos: leer, escribir y comentar en el aula. $13^{\mathrm{a}}$ edic. Barcelona, España: Paidós Ibérica.

Castelló, M. (Coord.) (2007). Escribir y comunicarse en contextos científicos y académicos. Conocimientos y estrategias. $1^{\text {a }}$ edic. Barcelona, España: Graó.

Chartier, A. M. (2004). Enseñar a leer y escribir Una aproximación histórica, $1^{a}$ edic. México D.F: Fondo de Cultura Económica.

Colom, A. (2006). “Texto, multimedialidad y sociedad del conocimiento. Consecuencias para la nueva educación", en Escolano, A. (ed.). Currículum editado y sociedad del conocimiento (pp. 35-55). Valencia, España: Tirant Lo Blanch.

Escolano, A. (Ed.) (2006). Currículum editado y sociedad del conocimiento. Texto, multimedialidad y cultura de la escuela. $1^{\text {a }}$ edic. Valencia, España: Tirant Lo Blanche.

Figueroa, B.; Aillon, M.; Salazar, O. (2012). "Avances hacia la comprensión del fenómeno de la alfabetización académica hipertextual en el contexto de la formación docente". Universum. Revista de Humanidades y Ciencias Sociales, 27(1), 55-70.

Gunnarsson, B.-L. (2003). "Genere-Based Pedagogies: A Social Response to Process". Journal of Second Language Writing, 12, 17-29.

Halliday, M. y Santana, J. (1982). El lenguaje como semiótica social: la interpretación social del lenguaje, del significado. 2a edic. México, D.F: Fondo de Cultura Económica.

Iser, W. (2005). Rutas de la interpretación. $1^{\mathrm{a}}$ edic. en español. México DF: Fondo de Cultura Económica.

Kress, G. (2003). El alfabetismo en la era de los nuevos medios. $1^{\text {a }}$ edic. española. Andalucía, España: Aljibe. 
Kristeva, J. (1969). "La palabra, el diálogo y la novela”, en Semiótica 1, vers. española, pp. 187- 225.

Marton, F. y Booth, S. (1997). Learning and awareness. $1^{\text {a }}$ ed. Mahwah, New Jersey, EE.UU.: Lawrence Erlbaum Associates, Inc.

Marton, F. y Saljö, R. (1976). "On qualitative differences in learning: I outcome and process". British journal of Educational Psychology, 46, 4-11.

Pardo, N. (2007). Cómo hacer análisis crítico del discurso. $1^{\text {a }}$ edic. Santiago, Chile: Frasis.

Piscitelli, A. (2005). Internet la imprenta del siglo XXI. $1^{\text {a }}$ edic. Barcelona, España: Gedisa S.A.

Wertsch, J. (1993). Voces de la mente. 1ª edic. Madrid, España: Visor. 